\title{
Respuesta clínica completa postneoadyuvancia en cáncer de recto estadio III: observación o cirugía
}

\section{Complete Clinical Response following Neoadjuvant Treatment of Stage II Rectal Cancer: Observation of Surgery}

Pilar Adriana Torres-Mesa, MD. ${ }^{1}$

Fellow Cirugía Gastrointestinal Oncológica y Endoscopia Digestiva. Instituto Nacional de Cancerología, Universidad Militar Nueva Granada. Bogotá, Colombia

E-mail: piadriana78@gmail.com

\begin{abstract}
Resumen
La respuesta clínica completa posterior a la terapia neoadyuvante del cáncer localmente avanzado de recto, se ha considerado suficiente para la implementación de una estrategia no quirúrgica, de observación y seguimiento por algunos autores. El manejo estándar de esta patología es la realización de manejo quirúrgico oncológico radical del tumor primario, 6 a 10 semanas posterior a la finalización de la neoadyuvancia. En esta revisión se exponen los pros y contras de cada propuesta y se describen las implicaciones y recomendaciones de cada alternativa de manejo.
\end{abstract}

\section{Palabras clave}

Cáncer de recto, repuesta clínica completa, manejo no quirúrgico, terapia neoadyuvante.

\begin{abstract}
Complete clinical response after neoadjuvant therapy for locally advanced rectal cancer has been considered sufficient for implementation of a non-surgical approach of observation and monitoring by some authors. Standard management of this condition is radical resection of the primary tumor six to ten weeks after completion of neoadjuvant therapy. In this review the pros and cons of each proposal are presented, and implications and recommendations for each alternative are described.
\end{abstract}

\section{Keywords}

Rectal cancer, complete medical response, nonsurgical management, neoadjuvant therapy

\section{INTRODUCCIÓN}

El cáncer colorrectal es la tercera patología oncológica más frecuente a nivel mundial y la segunda en EE.UU. y Europa (1). En Colombia ocupa el quinto lugar en la mortalidad general por cáncer, con una tasa de 5,3 por 100.000 habitantes, con un incremento anual promedio de 1,9 a $2,2 \%$ (2). En el Instituto Nacional de Cancerología en Bogotá, se reportó una incidencia de $3 \%$ en hombres y $2,1 \%$ en mujeres para el año 2010, siendo la histología de adenocarcinoma clásico en $73 \%$ de los casos, mucinoso en 5,2\% y $4,5 \%$ de variedad escamocelular (3).
El manejo se determina después de una estricta y precisa estadificación, de acuerdo con el TNM ( $7^{\text {th }}$ edición de la American Joint Comission on Cancer) (4). Para el cáncer de recto localmente avanzado, localizado en el recto medio o inferior (hasta $12 \mathrm{~cm}$ del margen anal), el tratamiento comprende una serie de pasos que son necesarios para el manejo integral de esta patología. El proceso requiere neoadyuvancia con quimioterapia y radioterapia, resección quirúrgica de la lesión (resección abdominoperineal o resección anterior baja de recto, con ostomía de protección o definitiva) y adyuvancia con quimioterapia (4-6). 
Después de completarse la quimiorradiación, entre la semana 6 a 10, se realiza una nueva estadificación y se determina en junta multidisciplinaria el tratamiento quirúrgico que requiere el paciente. Posteriormente, según los hallazgos patológicos del espécimen quirúrgico, el compromiso mesorrectal y ganglionar, el grado de regresión tumoral, si existe invasión linfovascular o perineural y la negativización de los bordes de resección, se determina la necesidad y protocolo de adyuvancia $(4,7,8)$.

La radioterapia se prefiere administrarla con intención neoadyuvante, porque la toxicidad es menor que cuando se administra después del procedimiento quirúrgico y se ha visto que genera esterilización de los tejidos mesorrectales, lo que en teoría garantiza una mejor calidad del procedimiento quirúrgico oncológico $(9,10)$.

\section{OBSERVACIÓN Y SEGUIMIENTO}

De 26 a 30\% de los tumores localmente avanzados de recto presentan respuesta clínica completa posterior a la quimiorradiación $(11,12)$. El primer grupo que reportó estos eventos, a cargo de la doctora Angelita Habr-Gama, propone una estrategia de seguimiento y observación para este tipo de pacientes, obviando el tratamiento quirúrgico de esta patología (12). Sin embargo, también existen detractores de esta opción de manejo (13) y por eso se exponen a continuación los pros y contras de cada estrategia.

Se define respuesta clínica completa cuando no hay evidencia de tumor rectal al examen clínico, endoscópico o imagenológico, durante la reestadificación de un paciente con cáncer localmente avanzado de recto, quien finalizó la terapia neoadyuvante. La respuesta patológica completa es la ausencia de lesión tumoral en el estudio histológico del espécimen quirúrgico (yTONOM0), resultado de una resección oncológica radical.

\section{A FAVOR}

La doctora Habr-Gama, con su grupo de la División de Cirugía Colorrectal, de la Escuela de Medicina de la Universidad de Sao Paulo, Brasil, fue la primera en describir pacientes con respuesta clínica completa después de la quimiorradiación con intención neoadyuvante, administrada a pacientes con cáncer localmente avanzado de recto y en proponer una estrategia de manejo no quirúrgico $(14,15)$.

En su primera serie (15) describe 265 pacientes con adenocarcinoma de recto distal (0-7 $\mathrm{cm}$ del margen anal) considerados resecables, quienes fueron evaluados entre 1991 y 2002. De ese grupo, 71 casos presentaron respuesta clínica completa, evaluada 8 semanas después de terminar la quimiorradiación $(26,8 \%)$ y fueron incorporados en un plan de seguimiento y observación. Los restantes 194 pacientes presentaron respuesta incompleta y fueron llevados a manejo oncológico radical. De este último grupo, 22 pacientes presentaron respuesta patológica completa y se compararon con el grupo observacional. Se identificó que el tamaño inicial de la lesión tumoral, el género, edad, diferenciación histológica del tumor y estadio clínico inicial, no mostraban significancia estadística entre ellos. Del grupo de observación se reportan 2 casos con recaída local, a los 56 y 64 meses de finalizar la neoadyuvancia. Fueron manejados con resección local y braquiterapia, sin mortalidad reportada hasta el final de la serie. Se presentaron 3 casos de recaída sistémica a los 18, 48 y 90 meses del seguimiento, que fueron manejados con quimioterapia sistémica. La tasa total de recurrencia fue de 7\% (5 pacientes). En el grupo quirúrgico con respuesta patológica completa, se reportaron 3 recaídas sistémicas $(13,6 \%)$ antes de completar 2 años de seguimiento, con desenlaces fatales en 2 de ellos.

En la siguiente serie de la doctora Habr-Gama, con 360 pacientes, ratifica la incidencia de complicaciones perioperatorias y los beneficios del manejo observacional (16).

Los puntos a considerar en la implementación de esta estrategia de manejo son:

- Menor morbilidad de la radioterapia cuando se administra con intención neoadyuvante, que posterior a cualquier procedimiento quirúrgico (17).

- La cirugía oncológica radical del cáncer del tercio distal, es un procedimiento mutilante, que requiere ostomía definitiva y que presenta significante mortalidad y morbilidad.

- La morbilidad del procedimiento oscila entre 26 y $45 \%$.

- Cuando se realiza resección anterior baja de recto, en tumores del tercio medio o anastomosis coloanal en tumores del recto inferior, la presentación de filtración anastomótica oscila entre 10 y $25 \%$ de los casos.

- La realización de una anastomosis coloanal puede generar tasas de incontinencia fecal hasta de $20 \%$, siendo mayor en los pacientes con radioterapia previa.

- La disfunción sexual y urinaria, como complicación del procedimiento quirúrgico, pueden ocurrir hasta en $50 \%$ de los casos, aun realizándose meticulosos procedimientos de preservación de la inervación pélvica, en centros altamente especializados (18).

- La lenta cicatrización de la región perianal posterior a resección abdominoperineal en pacientes con radioterapia pélvica, genera importante morbilidad local (17).

- Las tasas de mortalidad del manejo quirúrgico oncológico del cáncer de recto, oscilan entre 5 y $10 \%$.

- Las complicaciones del estoma (hernia parostomal, infección, prolapso) se presentan en 2 a $7 \%$ de los casos.

- No se reportaron beneficios en las tasas de supervivencia libre de enfermedad o global de los pacientes con 
manejo quirúrgico, respecto a los del manejo observacional.

- La ansiedad del cirujano o del tratante disminuye con la implementación del manejo oncológico estándar, que con la estrategia de seguimiento.

Weisser y su grupo reportan que la recurrencia local después del manejo observacional, ocurre entre las semanas 12 a 18 y puede ser susceptible aún de manejo quirúrgico. Además, determina que la supervivencia libre de enfermedad de pacientes tratados quirúrgicamente después de respuesta clínica completa, es de 85 a $90 \%$, si concomitantemente presentaban respuesta patológica completa (11).

La estadificación clínica inicial no se relacionó con recurrencia de la enfermedad y los pacientes con recaída sistémica tenían significantemente baja evidencia radiológica de metástasis nodales, sugiriendo que esta se desarrolla por diseminación hematógena más que linfática (18). Además, la presentación de ese tipo de recaída es más temprana que la endoluminal y por eso varios grupos han sugerido la adición de quimioterapia adyuvante o de inducción, después de la respuesta clínica completa, para limitar la presentación de ese tipo de recaída (19).

En el 2010, la doctora Habr-Gama describe los patrones clínicos y endoscópicos que determinan una respuesta clínica completa posquimiorradiación, de casos tratados con cáncer localmente avanzado de recto (20). Para diagnosticarla se debe encontrar:

- Aclaramiento de la mucosa en el área de la pared rectal comprometida.

- Telangiectasias o discreta vascularización sobre la mucosa rectal afectada previamente por el tumor.

- Pérdida de la elasticidad de la pared rectal, con sensación de rigidez o discreta cicatriz local. Debe diferenciarse de una estenosis o nodularidad de la mucosa, que correspondería a una respuesta clínica incompleta

- No visualización ni palpación de tumor, sobre una zona rectal donde previamente existía una masa.

Adicionalmente debe existir ausencia de enfermedad residual, es decir, sin evidencia de ulceración de la mucosa, irregularidad evidente, nódulo palpable, masa, estenosis o friabilidad local. La toma de biopsias de las lesiones o la cicatriz del sitio de la tumoración previa, no descarta la presencia de enfermedad residual y deben tomarse a discreción del tratante, sin dejar de considerar la posibilidad de una resección local o quirúrgica radical, si existen dudas o sospecha de respuesta incompleta $(21,22)$.

Se ha determinado que existe mayor posibilidad del hallazgo de respuesta clínica completa, si la estadificación postneoadyuvancia se realiza después de la semana 6 , gene- rando una mayor consideración de la estrategia del manejo observacional (23).

La microcirugía endoscópica transanal (TEM) debe restringirse a pacientes con tumores rectales confinados a la pared después de la quimiorradiación y que presentan ausencia de patrones patológicos desfavorables (ypT1, regresión tumoral completa) (24).

En conclusión, aunque existe el riesgo de presentarse una recaída local en los primeros 12 meses del seguimiento, la regularidad y frecuencia de esta hace que el tratamiento de salvamento se desarrolle rápidamente. Si se genera un concienzudo y estricto seguimiento de los pacientes con respuesta clínica completa postneoadyuvancia, puede evitarse la morbimortalidad de un procedimiento quirúrgico radical (25).

\section{EN CONTRA}

La estrategia de "wait and see" es contraria a los tradicionales principios quirúrgicos del manejo del cáncer de recto, donde se contempla la realización de resección oncológica radical del tumor primario, después de la neoadyuvancia.

Bonnetain y su grupo, en el metanálisis que comparaba los datos de pacientes de la EORTC 22921 (European Organisation for Research and Treatment of Cancer) con 1.011 pacientes y la FFCD 9203 (Fédération Francophone de Cancérologie Digestive) con 756 pacientes, dos estudios randomizados fase III, sobre la neoadyuvancia en el cáncer localmente avanzado de recto, comparan la quimiorradiación con la radioterapia sola. Reporta que la concomitancia del 5-fluorouracilo (5-FU) con la radioterapia preoperatoria aumenta las tasas de respuesta clínica y patológica completa, optimizando el control locoregional, por la esterilización de los depósitos mesorrectales y la disminución del volumen tumoral, sin evidenciarse aumento de la supervivencia libre de enfermedad o global (26).

Glynne-Jones y su grupo, en una revisión de 218 estudios fase I/II y 28 estudios fase III, determinan la efectividad y racionalidad de la política de "wait and see", ratificando el concepto que en algunos pacientes esa estrategia de manejo no es conveniente (13). Considera que los siguientes puntos deben tenerse en cuenta antes de tomar esa decisión:

- No existió uniformidad de la definición de respuesta clínica completa ni por qué medios se describía (imágenes diagnósticas, examen clínico o digital rectal, endoscopia y biopsia, todos los anteriores).

- Ningún estudio utilizó los criterios RECIST para evaluar el tamaño tumoral o compromiso regional.

- Se encontró que 15 a $25 \%$ de pacientes con respuesta patológica completa (ypT0), también presentaban ganglios linfáticos positivos. Es suficiente considerar que 
esas adenopatías están fuera del área de irradiación y ¿con ajustarla se logra un mayor control regional? ¿La morbilidad de la radioterapia justifica esta conducta?

- La verdadera significancia clínica de esos focos residuales microscópicos de enfermedad y si esas células pueden ser viables de tumor en el mediano o largo plazo es uno de los riesgos que se evitan con el manejo oncológico radical.

- La recurrencia del cáncer de recto después de que ha recibido manejo inicial con radioterapia tiene una presentación pélvica regional de comportamiento difuso, con el riesgo inherente de recaída a distancia, principalmente hepática. Esto genera que las opciones quirúrgicas ante la recaída sean más agresivas y en ocasiones no viables.

- La radiosensibilidad intrínseca o relación de dosis de radioterapia para el cáncer de recto es significantemente alta y la respuesta a ella es menor en tumores T3, $\mathrm{T} 4$, fijos o adheridos a planos profundos.

- Los datos publicados por la doctora Habr-Gama no han sido duplicados en ninguna unidad y no son enteramente consistentes. Lo anterior por considerar casos de tumores T2N0 dentro de sus series.

- Las técnicas de imágenes y de estadificación actual pueden generar una sobreestimación clínica inicial de tumores tempranos y por eso se ven esos aparentes resultados de respuesta clínica completa después de la neoadyuvancia (27).

- Además, las actuales técnicas de imágenes son poco confiables en la estadificación tumoral postneoadyuvancia, dada la baja correspondencia entre la predicción del status patológico y la actual evaluación del espécimen quirúrgico (28).

- Todos los pacientes con recurrencia local después de quimiorradiación, murieron dentro de los tres años siguientes.

- Puede haber argumentos para su implementación en tumores tempranos $\mathrm{T} 1 / \mathrm{T} 2$, de pacientes ancianos o con comorbilidades (29-31).

- La evidencia disponible es insuficiente para adoptar esa política en un paciente joven, sin comorbilidades o riesgo quirúrgico bajo, para el manejo integral de su patología (32-35).

Rullier y su grupo (36) generaron una clasificación quirúrgica del cáncer rectal del tercio distal, sugiriendo la realización de una cirugía preservadora de esfínteres sin comprometer la morbilidad, control local pélvico o la supervivencia. Lo anterior se justifica en términos de mantener la imagen corporal del paciente, la función genital y calidad de vida, sin sacrificar el manejo oncológico radical de esta patología (37).
Se concluye que debe mantenerse una actitud de reserva y precaución hacia la estrategia observacional, que representa una decisión de retardar o evitar la realización de una resección radical curativa, sin la disponibilidad de datos confiables o protocolos prospectivamente definidos (38-40).

\section{IMPLICACIONES Y RECOMENDACIONES DE LAS ALTERNATIVAS}

- Puede inferirse que la respuesta a la quimiorradiación con intención neoadyuvante es solo un factor pronóstico que nos ayuda a diferenciar pacientes de alto y bajo riesgo de recurrencia, para determinar quiénes pueden ser curados con una resección local o si requieren cirugía radical.

- En pacientes de edad avanzada, con tumores tempranos del tercio distal del recto cT1 o cT2, sin posibilidades de resección local, alto riesgo quirúrgico y comorbilidades importantes que contraindiquen la realización de una resección oncológica radical, la estrategia de observación y seguimiento es una opción viable.

- Para ello se requiere de parte del gastroenterólogo, cirujano colorrectal o gastrointestinal, que sea líder del seguimiento del paciente, no solo un claro entendimiento de las implicaciones y regularidad del examen físico, sino también de los componentes del mismo.

- El seguimiento requiere la realización de un examen físico completo, examen digital rectal, rectoscopia o rectosigmoidoscopia cada 1 a 3 meses según los protocolos institucionales a discreción del tratante. Si hay lesión, ulcera, nódulo o masa se recomienda la toma de biopsias. Aunque ellas no descarten la presencia de lesión maligna, es pieza útil de documentación de la recaída, si se presenta.

- La toma de imágenes diagnósticas se recomienda que sea cada 6 meses. También la realización de RNM pélvica para evaluar la región mesorrectal y ganglionar pélvica, el compromiso de la pared rectal e infiltración a estructuras vecinas y el TAC de tórax y abdomen, para descartar la enfermedad sistémica. La ultrasonografía rectal no se considera de elección para el seguimiento, por la alteración de las ecocapas de la pared rectal por la radioterapia. No existen estudios que recomienden rutinariamente la toma de PET-CT.

- Con cada consulta o examen físico, se recomienda la toma de bioquímica sanguínea, con perfil hepático y toma de antígeno carcinoembrionario y Ca 19-9.

- El paciente debe entender claramente que existe riesgo de recurrencia de la enfermedad, que en su mayoría se presenta endoluminal, pero que puede darse regional (mesorrectal, ganglionar o peritoneal) y sistémica (pul- 
monar, hepática, ósea). Debe firmarse consentimiento informado para esta estrategia de manejo y dejarse consignado todo lo anterior en la historia clínica.

- También el paciente debe conocer el manejo quirúrgico y oncológico de cada recaída, que puede ser con resección local si es endoluminal, resección radical oncológica (resección abdominoperineal con ostomía definitiva, resección anterior baja de recto con ostomía temporal o solo ostomía derivativa si es irresecable). Según el caso, puede ser necesaria la adyuvancia con quimioterapia o medicamentos biológicos.

- Si se decide la realización de resección local transanal o resección oncológica radical, deben entenderse las complicaciones, beneficios y consideraciones de cada alternativa por parte del paciente, incluyendo la posibilidad o requerimiento de una ostomía definitiva, quimioterapia adyuvante según el reporte de la patología final y que el seguimiento es una pieza clave del tratamiento.

En conclusión, la comunicación y entendimiento de las implicaciones del manejo observacional o quirúrgico escogido, por parte del personal médico y del paciente, genera la adherencia y confianza de ambas partes para un adecuado seguimiento clínico, endoscópico e imagenológico, que permita el diagnóstico precoz y tratamiento de la recaída de la enfermedad. También que estos pacientes puedan evaluarse de manera prospectiva, con miras a la realización de estudios clínicos que ofrezcan las respuestas aún sin responder sobre el tema.

\section{REFERENCIAS}

1. Siegel R, Naishadham D, Jemal A. Cancer statistics, 2013. Ca Cancer J Clin 2013; 63: 11-30.

2. Instituto Nacional de Cancerología E.S.E. Anuarios estadísticos 2003 - 2011. Bogotá.

3. Piñeros M, Pardo C, Gamboa O, et al. Atlas de mortalidad por cáncer en Colombia, tercera edición. Bogotá, 2010. Instituto Nacional de Cancerología E.S.E; Instituto Geográfico Agustín Codazzi.

4. National Comprehensive Cancer Network. NCCN Clinical Practice Guidelines in Oncology, Rectal Cancer, Version 2.2014. URL: http://www.nccn.org/professionals/physician_gls/f_guidelines.asp.

5. Van de Velde CJ, Boelens PG, Borras JM, et al. EURECCA colorectal: Multidisciplinary management: European consensus conference Colon \& Rectum. Eur J Cancer 2014; 50(1): 1.e1-1.e34.

6. Meredith KL, Hoffe SE, Shibata D. The multidisciplinary management of rectal cancer. Surg Clin N Am 2009; 89: 177-215.

7. Glynne-Jones R, Kronfli M. Locally advanced rectal cancer: A comparison of management strategies. Drugs 2011; 71(9): 1153-1177.
8. Valentini V, Beets-Tan R, Borras JM, et al. Evidence and research in rectal cancer. Radiotherapy and Oncology 2008; 87: 449-474.

9. McCarthy K, Pearson K, Fulton R, Hewitt J. Quimiorradiación preoperatoria para el cáncer rectal localmente avanzado no metastásico. Cochrane Database of Systematic Reviews 2012 Issue 12. Art. No.: CD008368.

10. Jeong WL, Jong HL, Jun-Gi K, et al. Comparison between preoperative and postoperative concurrent chemoradiotherapy for rectal cancer: an institutional analysis. Radiat Oncol J 2013; 31(3): 155-161.

11. Weisser M, Beets-Tan R and Beets G. Management of complete response after chemoradiation in rectal cancer. Surg Oncol Clin N Am 2014; 23 (1): 113-125.

12. Habr-Gama A, M.D., Perez RO, Nadalin W. Long-term results of preoperative chemoradiation for distal rectal cancer correlation between final stage and survival. J Gastrointest Surg 2005; 9: 90-101.

13. Glynne-Jones R, Wallace M, Livingstone JIL, et al. Complete clinical response after preoperative chemoradiation in rectal cancer: Is a "wait and see" policy justified? Diseases of the Colon \& Rectum 2008; 51: 10-20.

14. Habr-Gama A, de Souza PM, Ribeiro U, et al. Low rectal cancer: impact of radiation and chemotherapy on surgical treatment. Dis Colon Rectum 1998; 41: 1087-1096.

15. Habr-Gama A, Perez RO, Nadalin W, et al. Operative versus nonoperative Treatment for Stage 0 Distal Rectal Cancer Following Chemoradiation Therapy. Long-term Results. Ann Surg 2004; 240:711-718.

16. Habr-Gama A, Perez RO, Nadalin W. Long-term results of preoperative chemoradiation for distal rectal cancer correlation between final stage and survival. J Gastrointest Surg 2005; 9: 90-101.

17. Habr-Gama A. Assessment and management of the complete clinical response of rectal cancer to chemoradiotherapy. Colorectal Disease 2006; 8(3): 21-24.

18. Habr-Gama A, Perez RO, Proscurshim I, et al. Patterns of failure and survival for nonoperative treatment of stage $\mathrm{c} 0$ distal rectal cancer following neoadjuvant chemoradiation therapy. J Gastrointest Surg 2006; 10: 1319-1329.

19. Habr-Gama A, Perez RO, São Julião GP, et al. Nonoperative approaches to rectal cancer: A critical evaluation. Semin Radiat Oncol 2011; 21: 234-239.

20. Habr-Gama A, Perez RO, Wynn G, et al. Complete clinical response after neoadjuvant chemoradiation therapy for distal rectal cancer: Characterization of clinical and endoscopic findings for standardization. Dis Colon Rectum 2010; 53: 1692-1698.

21. Habr-Gama A, Perez R, Proscurshim I, et al. Complete clinical response after neoadjuvant chemoradiation for distal rectal cancer. Surg Oncol Clin N Am 2010; 19: 829-845.

22. Perez RO, Habr-Gama A, Pereira GV, et al. Role of biopsies in patients with residual rectal cáncer following neoadjuvant chemoradiation after downsizing: can they rule out persisting cancer? Colorectal Disease 2011; 14: 714-720. 
23. Perez RO, Habr-Gama A, Sao Juliao GP, et al. Optimal Timing for Assessment of Tumor Response to Neoadjuvant Chemoradiation in Patients with Rectal Cancer: Do All Patients Benefit from Waiting Longer than 6 Weeks? Int J Radiat Oncol Biol Phys 2012; 84(5): 1159-1165.

24. Perez RO, Habr-Gama A, Lynn PB, et al. Transanal Endoscopic Microsurgery for Residual Rectal Cancer (ypT0-2) Following Neoadjuvant Chemoradiation Therapy: Another Word of Caution. Dis Colon Rectum 2013; 56: 6-13.

25. Habr-Gama A, Sabbaga J, Gama-Rodrigues J, et al. Watch and Wait Approach Following Extended Neoadjuvant Chemoradiation for Distal Rectal Cancer: Are We Getting Closer to Anal Cancer Management? Dis Colon Rectum 2013; 56: 1109-1117.

26. Bonnetain F, Bosset JF, Gerard JP, et al. What is the clinical benefit of preoperative chemoradiotherapy with 5FU/leucovorin for T3-4 rectal cancer in a pooled analysis of EORTC 22921 and FFCD 9203 trials: Surrogacy in question?. European Journal of Cancer 2012; 48 (12): 1781-1790.

27. O’Neill BP, Brown G, Heald RJ. Non-operative treatment after neoadjuvant chemoradiotherapy for rectal cancer. Lancet Oncol 2007; 8: 625-633.

28. De Nardi P, Carvello M. How reliable is current imaging in restaging rectal cancer after neoadjuvant therapy? World J Gastroenterol 2013; 19(36): 5964-5972.

29. Hughes R, Harrison M, Glynne-Jones R. Could a wait and see policy be justified in T3/4 rectal cancers after chemoradiotherapy? Acta Oncológica 2010; 49: 378-381.

30. Dalton RSJ, Velineli R, Osborne ME, et al. A single centre experience of chemoradiotherapy for rectal cancer: Is there potential for nonoperative management? Colorectal Disease 2011; 14: 567-571.

31. Lim L, Chao M, Shapiro J, et al. Long-Term Outcomes of Patients with Localized Rectal Cancer Treated with Chemoradiation or Radiotherapy Alone Because of Medical
Inoperability or Patient Refusal. Dis Colon Rectum 2007; 50: 2032-2039.

32. Neuman HB, Elkin EB, JG Guillem, et al. Treatment for Patients with Rectal Cancer and a Clinical Complete Response to Neoadjuvant Therapy: A Decision Analysis. Dis Colon Rectum 2009; 52 (5): 863-871.

33. Mignanelli ED, Campos-Lobato LF, Stocchi L, et al. Down staging after chemoradiotherapy for locally advanced rectal cancer: Is there more (tumor) than meets the eye? Diseases of the Colon \& Rectum 2010; 53: 251-256.

34. Higgins KA, Willett CG, Czito BG. Nonoperative management of rectal cancer: Current perspectives. Clinical Colorectal Cancer 2010; 9(2): 83-88.

35. Maas M, Nelemans PJ, Valentini V, et al. Long-term outcome in patients with a pathological complete response after chemoradiation for rectal cancer: a pooled analysis of individual patient data. Lancet Oncol 2010; 11: 835-44.

36. Rullier E, Denost Q, Vendrely V, et al. Low Rectal Cancer: Classification and Standardization of Surgery. Dis Colon Rectum 2013; 56: 560-567.

37. Smith FM, Waldron D, Winter DC. Rectum-conserving surgery in the era of chemoradiotherapy. Br J Surg 2010; 97:1752-1764.

38. Nyasavajjala SM, Shaw AG, Khan AQ et al. Neoadjuvant chemo-radiotherapy and rectal cancer: can the UK watch and wait with Brazil? Colorectal Disease 2010; 12: 33-36.

39. Fischkoff KN, Ruby JA, Guillem JG. Nonoperative approach to locally advance rectal cancer after neoadjuvant combined modality therapy: Challenges and opportunities from a surgical perspective. Clinical Colorectal Cancer 2011; 10(4): 291-7.

40. Martin ST, Heneghan MH, Winter DC. Systematic review and meta-analysis of outcomes following pathological complete response to neoadjuvant chemoradiotherapy for rectal cancer. British Journal of Surgery 2012; 99: 918-928. 